\title{
EXISTENCE AND UNIQUENESS OF FUNCTIONAL CALCULUS HOMOMORPHISMS
}

\author{
BY WILLIAM R. ZAME ${ }^{1}$
}

Communicated by Chandler Davis, July 19, 1975

The purpose of this note is to announce a uniqueness result for the holomorphic functional calculus in commutative Banach algebras which is much stronger than the usual uniqueness assertion.

Let $A$ be a commutative Banach algebra with unit and let $\mathrm{a}=\left(a_{1}, \ldots\right.$, $a_{n}$ ) be an $n$-tuple of elements of $A$. Denote by $\sigma(a)$ the joint spectrum of a and by $O(\sigma(a))$ the topological algebra of germs of functions holomorphic near $\sigma(a)$. The holomorphic functional calculus, developed by Shilov [6] , Arens-Calderón [4] and Waelbroeck [7], provides a continuous unital homomorphism $\theta_{\mathrm{a}}$ : $O(\sigma(a)) \rightarrow A$ such that:

(i) $\theta_{\mathrm{a}}\left(z_{i}\right)=a_{i}$ for $i=1,2, \ldots, n$,

(ii) $\theta_{\mathrm{a}}(f)^{\wedge}=f \circ\left(\hat{a}_{1}, \ldots, \hat{a}_{n}\right)$ for each $f$ in $O(\sigma(a))$,

where $\hat{b}$ denotes the Gelfand transform of an element $b$ of $A$, acting on $\Delta A$ (the maximal ideal space of $A)$. If $\mathrm{a}^{\prime}=\left(a_{1}, \ldots, a_{n}, a_{n+1}, \ldots, a_{n+m}\right)$ is an $n+m$ tuple and $\pi: \mathbf{C}^{n+m} \rightarrow \mathbf{C}^{n}$ is the projection, then the homomorphisms $\theta_{\mathrm{a}}$ and $\theta_{\mathrm{a}^{\prime}}$ satisfy the following compatibility condition:

(iii) $\theta_{\mathrm{a}}(f)=\theta_{\mathrm{a}}(f \circ \pi)$ for each $f$ in $O(\sigma(\mathrm{a}))$.

The usual uniqueness assertion is that the family $\left\{\theta_{\mathbf{a}}\right\}$ is unique subject to these requirements. However, we can show that the compatibility condition is redundant, and that the individual homomorphisms are themselves unique.

THEOREM 1. The homomorphism $\theta_{\mathrm{a}}$ is the unique continuous unital homomorphism of $O(\sigma(a))$ into $A$ which satisfies conditions (i) and (ii) above.

Theorem 1 follows as an application of a more general existence and uniqueness result (Theorem 2 , below). Let $U$ be a domain in $\mathbf{C}^{n}, \widetilde{U}$ its envelope of holomorphy and $O(U)$ the Fréchet algebra of holomorphic functions on $U$. Each holomorphic function $f$ on $U$ has a unique extension to $\widetilde{U}$, which we denote by $\widetilde{f}$. Note that each continuous unital homomorphism $\phi: O(U) \rightarrow A$ with $\phi\left(z_{i}\right)=a_{i}$ for $i=1, \ldots, n$ has a continuous adjoint $\phi^{*}: \Delta A \rightarrow \widetilde{U}$ such that $\widetilde{z}_{i} \circ \phi^{*}=\hat{a}_{i}$ for $i=1, \ldots, n$. The following result shows that this correspondence of $\phi$ with $\phi^{*}$ is bijective.

AMS (MOS) subject classifications (1970). Primary $46 \mathrm{~J} 05$.

Key words and phrases. Functional calculus, Shilov-Arens-Caldero'n theorem.

${ }^{1}$ Supported in part by National Science Foundation Grant PO 37961-001. 
THEOREM 2. For each continuous function $\tau: \Delta A \rightarrow \widetilde{U}$ such that $\widetilde{z}_{i} \circ \tau$ $=\hat{a}_{i}$ for $i=1, \ldots, n$, there is a unique continuous unital homomorphism $\phi: O(U) \rightarrow A$ such that $\phi^{*}=\tau$ and $\phi\left(z_{i}\right)=a_{i}$ for $i=1, \ldots, n$.

As another application of Theorem 2, we obtain the following lifting result.

THEOREM 3. Let I be a closed ideal of $A$ which is contained in the radical and let $\eta: A \rightarrow A / I$ be the quotient map. Let $U$ be an open subset of $\mathbf{C}^{n}$ and let $\phi: O(U) \rightarrow A / I$ be a continuous unital homomorphism. For each $i=1$, $\ldots, n$, let $a_{i}$ be an element of $A$ with $\eta\left(a_{i}\right)=\phi\left(z_{i}\right)$. Then there is a unique continuous unital homomorphism $\bar{\phi}: O(U) \rightarrow A$ such that $\eta \circ \bar{\phi}=\phi$ and $\bar{\phi}\left(z_{i}\right)$ $=a_{i}$ for $i=1, \ldots, n$.

In [2] and [3], Allan obtains extensions of the usual functional calculus to Stein spaces, but he shows that the correspondence of holomorphic functions to elements of the algebra cannot always be chosen to be a homomorphism. The following analog of Allan's result shows that the difficulty in obtaining a homomorphism lies solely in the algebra of global functions.

Theorem 4. Let $X$ be a Stein space and let $\phi: O(X) \rightarrow A$ be a continuous unital homomorphism with adjoint $\phi^{*}: \Delta A \rightarrow X$. Then there is a continuous unital homomorphism $\widetilde{\phi}: O\left(\phi^{*}(\Delta A)\right) \rightarrow A$ such that $\widetilde{\phi}(f)=\phi(f)$ for each $f$ in $O(X)$.

The proofs utilize an infinite-dimensional functional calculus developed by Allan [1] and Craw [5]. Regarding $\Delta A$ as a compact subset of the weak-* dual of $A$, there is a natural homomorphism $\Theta: O(\Delta A) \rightarrow A$. We show that every continuous homomorphism $\phi: O(U) \rightarrow A$ factors through $\Theta$ and that the value of $\Theta(f)$, for $f$ in $O(\Delta A)$, depends only on the behavior of $f$ on a suitably small subvariety of a neighborhood of $\Delta A$. Details will appear elsewhere.

\section{REFERENCES}

1. G. R. Allan, A form of local characterization of Gelfand transforms, J. London Math. Soc. 43 (1968), 623-625. MR 37 \#6756.

2. - An extension of the Šilov-Arens-Calderón theorem, J. London Math. Soc. 44 (1969), 595-601. MR 39 \#4678.

3. - On lifting analytic relations in commutative Banach algebras, J. Functional Analysis 5 (1970), 37-43. MR 41 \#7437.

4. R. Arens and A. P. Calderón, Analytic functions of several Banach algebra elements, Ann. of Math. (2) 62 (1955), 204-216. MR 17, 177.

5. I. Craw, $A$ condition equivalent to the continuity of characters on a Fréchet algebra, Proc. London Math. Soc. (3) 22 (1971), 452-464. MR 44 \#4524. 
6. G. E. Šilov, On decomposition of a commutative normed ring in a direct sum of ideals, Mat. Sb. 32 (74) (1953), 353-364; English transl., Amer. Math. Soc. Transl. (2) 1 (1955), 37-48. MR 14, 884; 17, 512.

7. L. Waelbroeck, Le calcul symbolique dans les algèbres commutatives, J. Math. Pures Appl. (9) 33 (1954), 147-186. MR 17, 513.

DEPARTMENT OF MATHEMATICS, STATE UNIVERSITY OF NEW YORK AT BUFFALO, AMHERST, NEW YORK 14226

Current address: Department of Mathematics, Tulane University, New Orleans, 70118 